\title{
PHASE SEPARATION IN PM2000 Fe-BASE ODS ALLOY
}

\author{
C. Capdevila* and M. K. Miller** \\ * Centro Nacional de Investigaciones Metalúrgicas (CENIM-CSIC), Avda. Gregorio del Amo, 8; \\ Madrid, E-28040, Spain \\ ** Materials Science and Technology Division, Oak Ridge National Laboratory, P.O. Box 2008, \\ Oak Ridge, TN 37831-6136, USA
}

Coarse grained PM2000 is an Fe-based Oxide Dispersion Strengthened (ODS) steel formed by Mechanical Alloying (MA). This ODS alloy is a class of advanced materials offering both creepand oxidation-resistance and is used for tubing in a combined-cycle gas turbine (CCGT) heat exchanger in biomass power plants. PM2000 is an $\mathrm{Fe}-20$ at. \% $\mathrm{Cr}-5$ at.\% Al alloy to which yttria particles have been added to enhance creep performance. One goal of this study is to analyze whether this material exhibits the same hardening as high chromium stainless steels when heat treated at temperatures of $\sim 748 \mathrm{~K}\left(475^{\circ} \mathrm{C}\right)$. This hardening phenomenon has been termed $475^{\circ} \mathrm{C}$ embrittlement and has been attributed to phase separation into Fe-rich $\alpha$ and $\mathrm{Cr}$-rich $\alpha$ ' phases within the miscibility gap in the $\mathrm{Fe}-\mathrm{Cr}$ equilibrium system $[1,2]$.

The three-dimensional microstructure, as determined from atom probe tomography, resulting from phase separation and aging of the PM2000 alloy at $748 \mathrm{~K}$ is shown in Fig. 1. At this temperature and for the relatively low chromium content of the alloy, the Cr-rich $\alpha$ " phase is in the form of isolated particles rather than the interconnected network structure observed previously in $\mathrm{Fe}-45 \% \mathrm{Cr}$ alloys [2]. The kinetics of phase separation was quantitatively determined by analyzing the evolution of the geometrical domain size $(\lambda)$ and the composition amplitude $(\Delta C)$. The composition amplitude was determined from the composition frequency distribution with the Langer-Bar-on-Miller (LBM) method [3], and the scale from the 3D autocorrelation function [3]. The evolution of $\lambda$ and $\Delta C$ with aging time, $t$, at $748 \mathrm{~K}$ is shown in Fig. 2 and indicates that $\Delta C$ varies linearly with $\ln (t)$. Following the Lifshitz-Slyozov-Wagner (LSW) theory $[4,5]$, the evolution of scale $(\lambda)$ measured from the first minimum and the first maximum of the autocorrelation function with time were fitted to a power law. An exponent value of $0.32\left(\mathrm{R}^{2}=0.78\right)$ was obtained for the measurements of the first maximum, and a corresponding value of $0.34\left(\mathrm{R}^{2}=0.84\right)$ from the measurements of the first minimum. The relationship between $\lambda$ and $\Delta C$ and the hardness of this phase separated and aged PM2000 alloy is shown in Fig. 3. The hardness was found to increase linearly with increasing domain size, as shown in Fig. 3(a). The linear increase in composition amplitude with alloy hardness is shown in Fig. 3(b). In conclusion, the evolution of $\lambda$ can be fitted to a power law which is consistent with the power law growth of the mean precipitate size $\mathrm{R}(t)$ varying as $\sim t^{1 / 3}$ predicted by LSW theory [4-6].

[1] R. Lagnebor, Acta Metall. 15 (1967) 1737.

[2] M.K. Miller, et al., Acta Metall. Mater. 43 (1995) 3385.

[3] M. K. Miller, Atom Probe Tomography, Kluwer Academic / Plenum Press, New York, 2000, p. 28.

[4] I. M. Lifshitz and V. V. Slyozov, J. Phys. Chem. Solids 19 (1961) 35.

[5] C. Wagner, Z. Elektrochem. 65 (1961) 581.

[6] Research at the Oak Ridge National Laboratory SHaRE User Facility was sponsored by the Office of Basic Energy Sciences, Division of Scientific User Facilities, U.S. Department of Energy, under contract DE-AC0500OR22725 with UT-Battelle, LLC. 
(a)
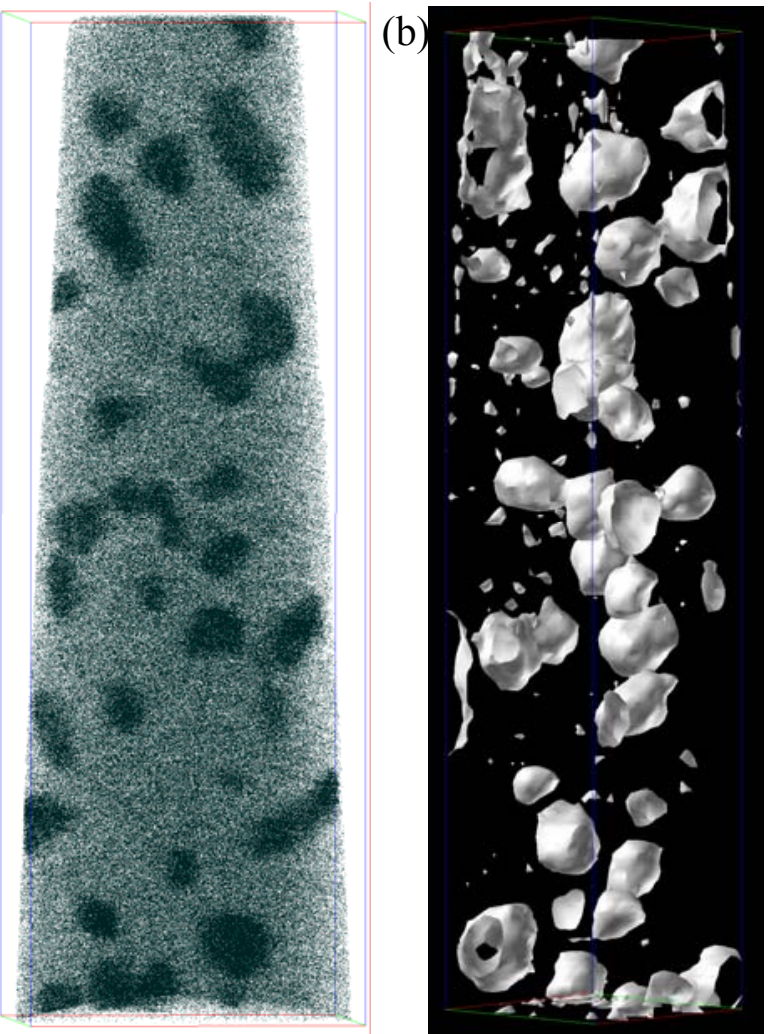

FIG 1. (a) $\mathrm{Cr}$ atom map and (b) $20 \%$ isoconcentration surface after $2040 \mathrm{~h}$ aging at $748 \mathrm{~K}$. At this late stage, the Cr-enriched $\alpha$ particles are isolated (volume $=30 \times 30 \times 127 \mathrm{~nm}$ ).

(a)



(a)

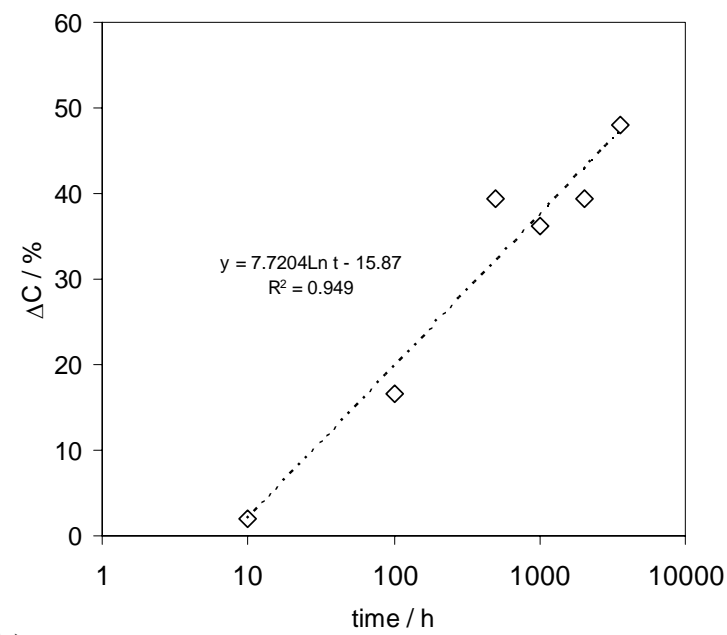

(b)

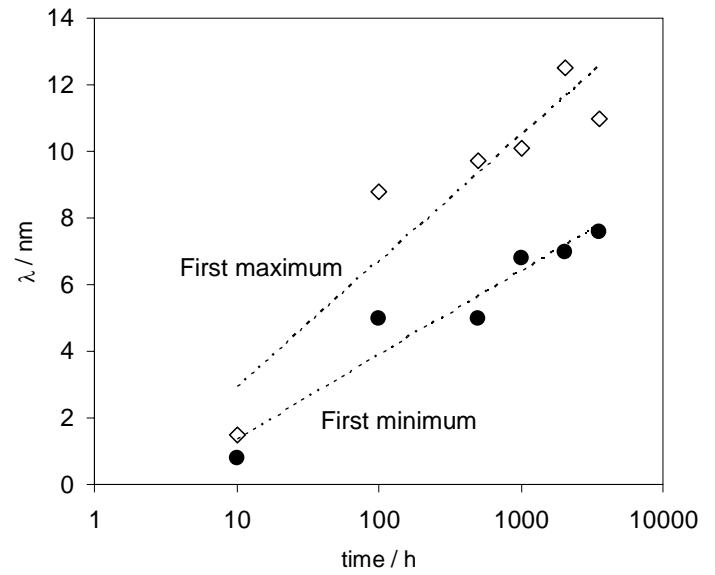

FIG 2. Evolution of (a) $\Delta C$ and (b) $\lambda$ with time

(b)

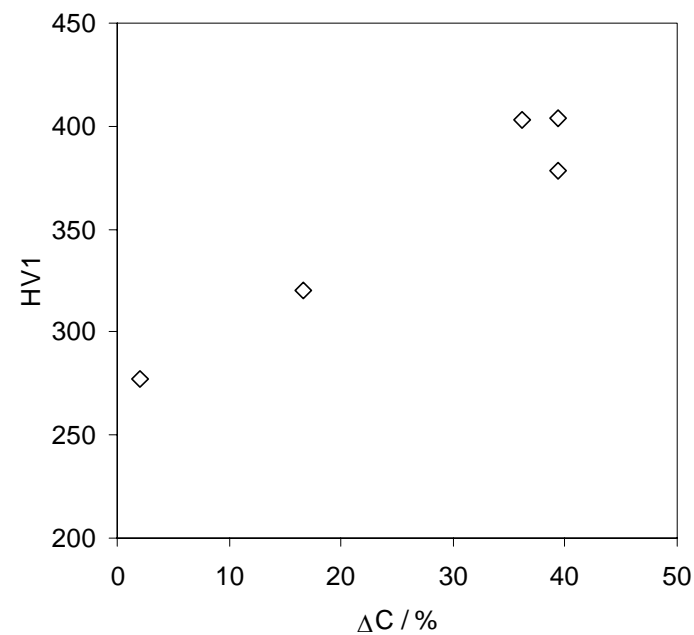

FIG 3. Relationship between hardness (HV1) and (a) scale of decomposition, and (b) composition amplitude 